This document is the accepted manuscript version of the following article:

Saccone, M., Hofhuis, K., Bracher, D., Kleibert, A., van Dijken, S., \& Farhan, A. (2020).

Elevated effective dimension in tree-like nanomagnetic cayley structures. Nanoscale, 12(1),

189-194. https://doi.org/10.1039/C9NR07510K

\title{
Elevated effective dimension in tree-like nanomagnetic Cayley struc-
} tures

\begin{abstract}
Michael Saccone, ${ }^{* a, b}$ Kevin Hofhuis, ${ }^{c, d}$, David Bracher $^{e}$, Armin Kleibert ${ }^{e}$, Sebastiaan van Dijken $^{b}$, and Alan Farhan*b,d

Using state-of-the-art electron-beam lithography, Ising-type nanomagnets may be defined onto nearly any two-dimensional pattern imaginable. The ability to directly observe magnetic configurations achieved in such artificial spin systems makes them a perfect playground for the realization of artificial spin glasses. However, no experimental realization of a finite-temperature artificial spin glass has been achieved so far. Here, we aim to get a significant step closer in achieving that goal by introducing an artificial spin system with random interactions and increased effective dimension: dipolar Cayley tree. Through synchrotron-based photoemission electron microscopy, we show that an improved balance of ferro- and antiferromagnetic ordering can be achieved in this type of system. This combined with an effective dimension as high as $d=2.72$ suggests that future systems generated out of these building blocks can host finite temperature spin glass phases, allowing for real-time observation of glassy dynamics.
\end{abstract}

\section{Introduction}

In magnetism, when interactions between magnetic moments are in conflict with each other, frustration arises. Systems incorporating such frustrated interactions are characterized by extensive degeneracies, exotic emergent phenomena, and non-trivial ground states 1 .4. Spin glasses have been one of the most prominent examples of frustrated magnetism. They usually consist of a jumble of competing ferro- and antiferromagnetic order, leading interactions to compete with one another $\frac{516}{6}$. Unlike other frustrated systems, the interaction between their spins are highly disordered. When competing interactions are sufficiently random, the system does not easily transition by single spin flips. Often a transition to a lower energy state requires a reconfiguration of a large portion of the system. This makes many states become metastable and the true ground state elusive. These complex energy landscapes and the associated behaviors, magnetic susceptibility cusps, difference in field cooled (FC) and zero field cooled (ZFC) magnetic moment, and non-uniform relaxation times, are all characteristic of spin glasses. The extensive degeneracy and nature of the ground state in spin glasses have been an area of extensive research over multiple decades, resulting in theoretical

\footnotetext{
a Physics Department, University of California, 1156 High Street, Santa Cruz, CA 95064, USA. E-mail: msaccone@ucsc.edu

${ }^{b}$ NanoSpin, Department of Applied Physics, Aalto University School of Science, P.O. Box 15100, FI-00076 Aalto, Finland. E-mail: alan.farhan@gmx.net

${ }^{c}$ Laboratory for Mesoscopic Systems, Department of Materials, ETH Zürich, 8093 Zürich, Switzerland.

${ }^{d}$ Laboratory for Multiscale Materials Experiments, Paul Scherrer Institute, 5232 Villigen PSI, Switzerland.

e Swiss Light Source, Paul Scherrer Institute, 5232 Villigen PSI, Switzerland.
}

spin glass models that became, among other examples, the basis of error-correcting codes ${ }^{7}$, problems of optimization ${ }^{8}$, and brain science paradigms 90 . Inspired by recent research on artificial frustrated systems ${ }^{11-28}$, a first step towards the realization of artificial Ising spin glasses was made recently 29. There, Ising-type single-domain nanomagnets are lithographically arranged onto Gaussian random sites and orientations with a controlled degree of disorder, the feature commonly believed to be the main ingredient in spin glasses. However, these Gaussian patterns of Isingtype nanomagnets, while exhibiting partial short-range order, fell short in accessing a typical spin glass phase, in part because of unbalanced competing ferro- and anti-ferromagnetic ordering. This raises several open questions, regarding the prospect of realizing an artificial spin glass system. For example, can a different system design be implemented that would better optimize the balance of competing interactions in such disordered systems, so that spin glass degeneracy might arise? Also, can this goal be achieved by designing structures that would exhibit a higher effective dimension 30 ?

Here, we aim to address these questions by defining dipolarcoupled Ising-type nanomagnets onto Cayley trees, finite portions of Bethe lattices (see Fig. 1). These structures bear three main advantages: First, their tree-like pattern increases the effective dimension 30 , which is linked to accesing spin glass behavior 31 . Second, the balance of competing ferro- and antiferromagnetic interactions between the magnetic moments can be better tuned compared to structures with a simple Gaussian-type disorder 29. Third, considering the complexity of the quest to achieve an artificial spin glass, these finite-size structures offer a comfortable building block approach in addressing this purpose. Such a 

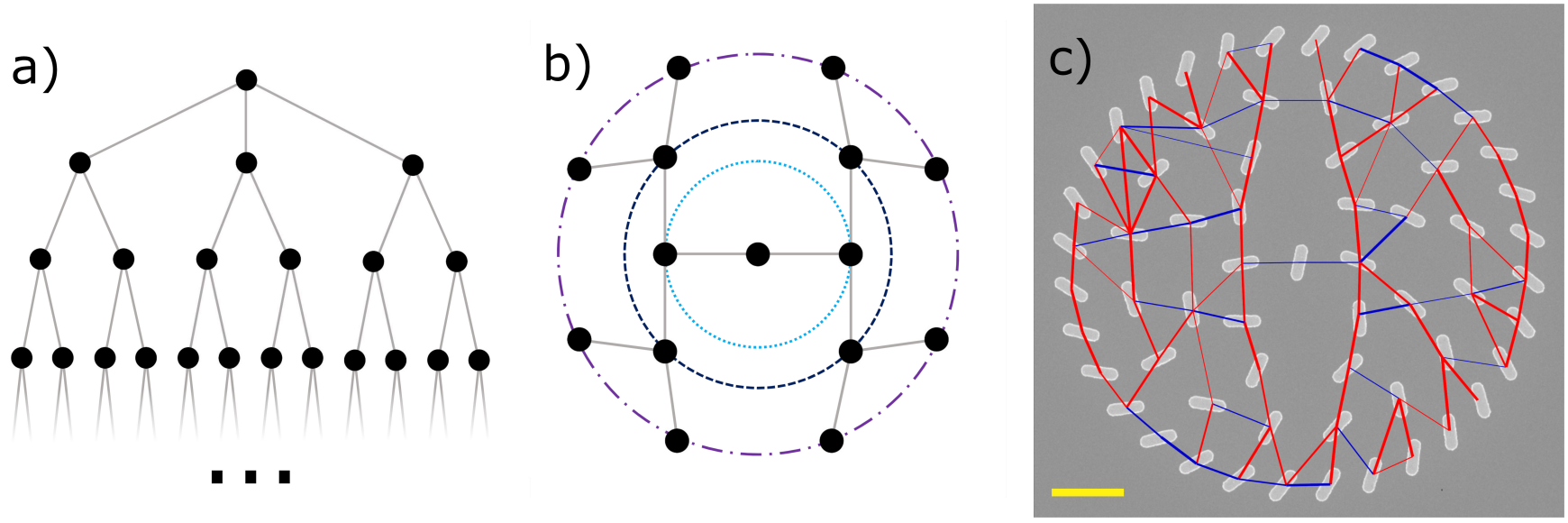

Fig. 1 (a) An illustration of a Bethe lattice with coordination number $k=3$. Black circles represent nodes connected by grey lines. (b) A Cayley tree modified to be embedded in two dimensions. Each circle represents a step taken from the center of the lattice. (c) Scanning electron microscope (SEM) image of a Cayley tree consisting of Ising-type nanomagnets with a length $L=300 \mathrm{~nm}$, width $W=100 \mathrm{~nm}$ and a thickness $t=2.6 \mathrm{~nm}$. The yellow scale bar corresponds to $600 \mathrm{~nm}$. The tree-like structure is best understood when looking at the inter-nanomagnet dipolar couplings, which are highlighted with red (ferromagnetic coupling) and blue lines (antiferromagnetic coupling) connecting centers of nanomagnets.

bottom-up approach has already been successfully applied to gain an understanding of ordering phenomena in the highly-frustrated artificial kagome spin ice 1132 . We begin with a methods section that describes our theoretical approach and how dipolar Bethe structures are designed, fabricated, and characterized using X-ray photoemission electron microscopy (X-PEEM). This is followed by a section where we describe the obtained results, starting from discussing moment configurations achieved after thermal annealing and the characterization of these ordering patterns in terms of spin correlations, effective dimension, and potential links to glassy behavior. We then conclude with a summary and outlook section where we assess the advantages of our Cayley structures as promising building blocks for the generation of the first artificial spin glass systems.

\section{Methods}

\subsection{Theoretical concept and design of dipolar Cayley trees}

Cayley trees are finite subset of infinite Bethe lattices. A Bethe lattice (see Fig. 19) is a tree-like graph with the helpful property that many models in statistical physics are exactly solvable on it 33 . To construct a Bethe lattice with a coordination number $k$, the number of connections per node, is chosen. $k$ nodes are connected to a central lattice site. From each of these nodes $k-1$ new nodes are attached, then $k-1$ additional nodes are attached from each of those. This continues ad infinitum in the case of a Bethe lattice and to a finite number of layers for a Cayley tree. Fig. 11 shows an example where $k=3$. Multiple studies suggest a non-zero spin glass transition temperature could be obtained if spins would be

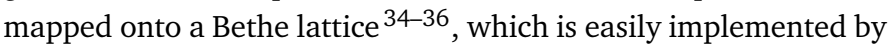
the patterning of Ising-type nanomagnets. To observe glassy dynamics, the spin glass transition temperature needs to be higher than the so-called blocking temperature $T_{B}$ of the patterned nanomagnets, as spin freezing occurs below $T_{B}$ 11]16]19|29|32.

Our nanomagnet samples mimic the essence of the Cayley tree's structure to create higher dimensional interaction networks. However, they differ from Cayley trees in three major ways. First, the spins interact via dipolar coupling. This increases the significance of next nearest neighbor effects, enabling the interaction network to leap from one branch to another. The finite-size nature of the fabricated Cayley trees serves as an advantage, as the increasing network density would cause nanomagnets to overlap and interactions between next nearest neighbors to grow. Finally, the central spin is only given two neighbors to reduce the eventual crowding at the sample boundary. Implementing these compromises, we design modified Cayley trees for embedding in two dimensions (see Fig. 13). The positions of the islands are uniformly spaced on concentric circles. The size of the circles is chosen to keep the distance of connected nodes constant (see grey lines in Fig. 1p). Here this sets the nearest neighbor distance to $a=500 \mathrm{~nm}$. The orientation of the magnets is chosen to create random interactions while simultaneously supporting the Bethe structure. To do so, one nanomagnet per circle has its orientation selected randomly from a uniform distribution. The members of the same circle should not interact with one another, to maintain the tree-like structure. Thus, their orientations are chosen to place them perpendicular to the dipolar magnetic field of their direct neighbors within each circle. Circles are added until nextnearest neighbor interactions begin to break down the tree-like structure, allowing for five circles in our systems. In Fig. 1c we show a typical structure and the network of interactions created by its geometry.

The illustrated network was formed by first considering the dipolar coupling strength between islands $i$ and $j$,

$$
J_{i j}=-\frac{\mu_{0}}{4 \pi}\left(\frac{\mathbf{S}_{i} \cdot \mathbf{S}_{j}}{r_{i j}^{3}}-3 \frac{\left(\mathbf{S}_{i} \cdot \mathbf{r}_{i j}\right)\left(\mathbf{S}_{j} \cdot \mathbf{r}_{i j}\right)}{r_{i j}^{5}}\right),
$$

where $\mu_{0}$ is the permittivity of free space, $\mathbf{S}_{i}$ is the magnetic moment of an island, and $\mathbf{r}_{i j}$ is the vector joining the center of two islands. If $J_{i j}$ is less than zero, the coupling is anti-ferromagnetic (blue lines in Fig. 1. F), and if it is higher than zero, the coupling is ferromagnetic (red lines in Fig. 1c). Where the magnitude of their dipole-dipole coupling was greater than $20 \%$ of maximum 
magnitude, two spins are considered connected. This threshold percentage represents a good approximation to relevant dipolar interactions, and results in networks that are neither too sparse nor too dense, to represent the effective dimension of the system. In either limit, the error of fit to find the effective dimension would grow substantially. Visualizing this network (see red and blue lines in Fig. 1c), a randomly modified tree-like network is apparent. The most prominent violations to pure tree-like structures occur in the outer ring as crowding increases the interaction strengths. Overall, we designed, fabricated and imaged 21 individual randomly generated Cayley structures using this theoretical concept. The interaction networks of these lattices are used to assess the effective dimension of the magnetic systems.

\subsection{Sample fabrication and XMCD imaging}

We used lift-off assisted electron-beam lithography to fabricate our samples (see Fig. 1). First, a 70-nm-thick layer of polymethylmethacrylate (PMMA) resist was spin-coated on top of a $1 \times 1$ $\mathrm{cm}^{2}$ silicon (100) substrate. Designed Cayley tree patterns are then exposed on the substrate using a VISTEC EBPG 5000PlusES system. A $2.6 \mathrm{~nm}$ permalloy $\left(\mathrm{Ni}_{80} \mathrm{Fe}_{20}\right)$ thin film was then evaporated at a base pressure of $3.6 \times 10^{-6} \mathrm{mbar}$, together with a $1.5 \mathrm{~nm}$ thick aluminum capping layer, to avoid fast oxidation of the structures. This is followed by a lift-off process in acetone, where all unwanted material is removed from top of the substrate. This results in Bethe structures consisting of nanomagnets with lengths $L=300 \mathrm{~nm}$ and widths $W=100 \mathrm{~nm}$. The size of the nanomagnets is chosen to ensure a mono-domain state and their elongated shape is set, so that the magnetization within each individual nanomagnet can only point in one of two possible direction along the long axis of the nanomagnet. Therefore, each individual nanomagnets represents a macroscopic Ising spin.


the nanomagnets is chosen so that the blocking temperature $T_{B}$ $=140-180 \mathrm{~K}$. With regard to XMCD imaging (see below), we define the blocking temperature as the temperature where magnetic moment re-orientations within the patterned nanomagnets start to occur at timescales equivalent to the time needed to record an XMCD image, which is about 7 seconds.

Following sample fabrication, and after a waiting period of three days in high vacuum at room temperature $(T=298 \mathrm{~K})$, the samples are transferred into a photoemission electron microscope (PEEM) 37 . There, the sample was cooled down to temperatures around $120 \mathrm{~K}$, ensuring that magnetic moments stay frozen within the timescale of hours, and magnetic imaging was performed employing x-ray magnetic circular dichroism (XMCD) at the $\mathrm{Fe} L_{3}$ edge $\frac{38}{38}$. The dark and bright contrast in a so-called XMCD image is a direct measure of the orientation of the magnetic moment of a nanomagnet relative to the x-ray polarization vector (see Fig. 2).

\section{Results}

\subsection{Spin-spin correlations and ordering preferences}

The relatively small size of these systems does not provide enough statistical relevance for a reliable extraction of typical or spin glass
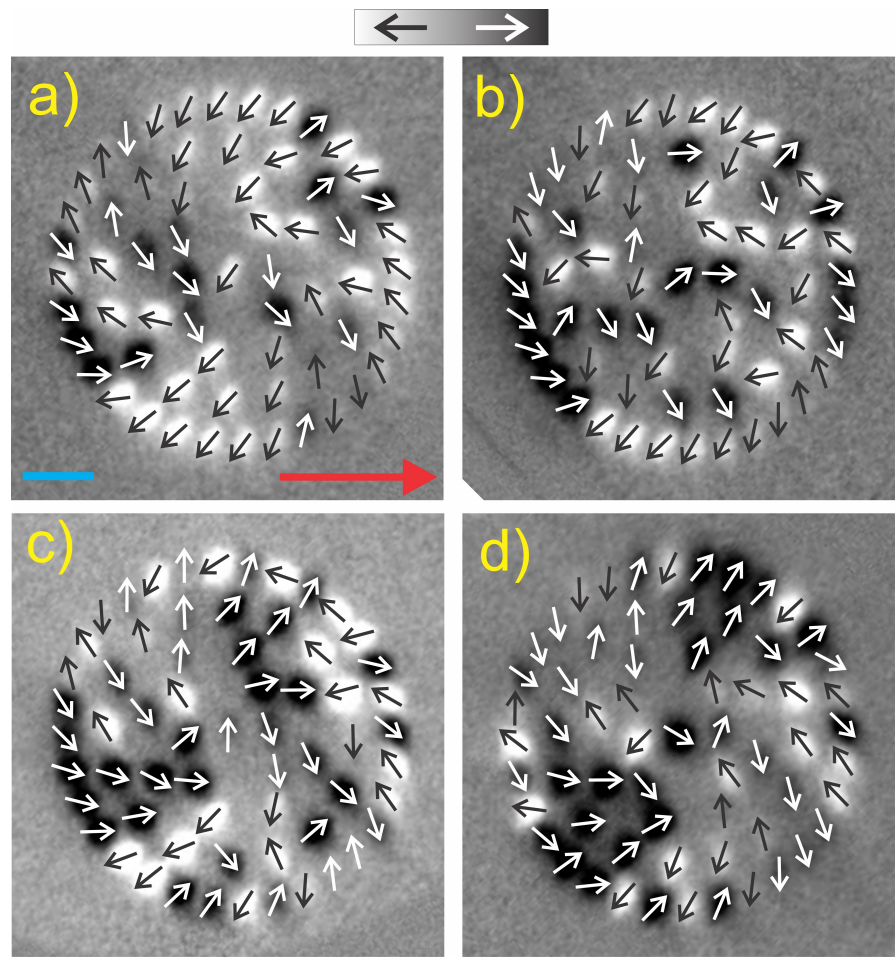

Fig. 2 (a)-(d) XMCD images of low-energy states achieved in four different Cayley tree structures, after thermal annealing. Moments pointing towards the incoming $\mathrm{x}$-rays (big red arrow) will appear dark, while moments opposing the incoming $x$-ray direction will appear bright. The blue bar indicates a length of $600 \mathrm{~nm}$.

correlation lengths. However, since our concern is equalizing antiferro- and ferromagnetic ordering, we focus on local correlations. Using a similar approach as in our most recent work ${ }^{29}$, we introduce a neighbor correlation function:

$$
[C]_{\mathrm{N}}=\frac{1}{N_{\text {pair }}} \sum_{i j}^{\mathrm{N}}\left\langle\Theta\left(\mathbf{S}_{i} \cdot \mathbf{S}_{j}\right)\right\rangle_{T} .
$$

$\Theta(x)=-1$ if $x$ is less than zero and $\Theta(x)=1$ otherwise, the sum was either taken over nearest neighbor (NN, $\left.r_{i j} \leq a\right)$, next-nearest neighbor (NNN, $r_{i j} \leq 2 a$ ), or next-next-nearest neighbor (NNNN, $r_{i j} \leq 3 a$ ) pairs, and $N_{\text {pair }}$ is the number of those pairs in a particular sum. This provides a consistent basis for measuring local correlations in these systems without translational invariance. A value below zero indicates predominantly antiferromagnetic coupling while one above zero indicates ferromagnetic coupling. These correlations are plotted for all systems of interest in Fig. $3 \mathrm{a}$.

A glance at these correlation measures reveals that nearestneighbor correlations in the Cayley tree structures have a weaker anti-ferromagnetic tendency when compared to structures with Gaussian disorder 29 (compare blue, black and red lines with dotted grey line in Fig 3 . .). There seems a tendency towards more ferromagnetic-type ordering in the Cayley tree structures, which can even be visually seen when looking at magnetic configurations shown in Fig. 2 through clusters of five to nine adjacent moments pointing in the same direction. Overall, the balance of ferro- and antiferromagnetic correlations is significantly im- 



Fig. 3 Neighbor correlations in randomized magnetic systems. In (a), the nearest-neighbor $(r / a=1)$, next-nearest-neighbor $(r / a=2)$, and nextnext-nearest neighbor $(r / a=3)$ correlations are plotted for the most ferromagnetically ordered Cayley tree system (red crosses), the average of all 21 Cayley tree systems (black triangles), the most anti-ferromagnetically ordered Cayley tree system (blue circles) and the most disordered system from a previously investigated artificial spin system with Gaussian disorder $\frac{29}{2}$ (grey squares). The green dotted line at $[\mathrm{C}]_{\mathrm{N}}=0$ represents balanced antiferro- and ferromagnetic correlation. (b) Moment configurations of the Cayley structure with the highest degree of ferromagnetictype ordering [red crosses and lines in (a)]. (c) Moment configurations of the Cayley structure with the strongest anti-ferromagnetic spin ordering [blue circles and lines in (a)].
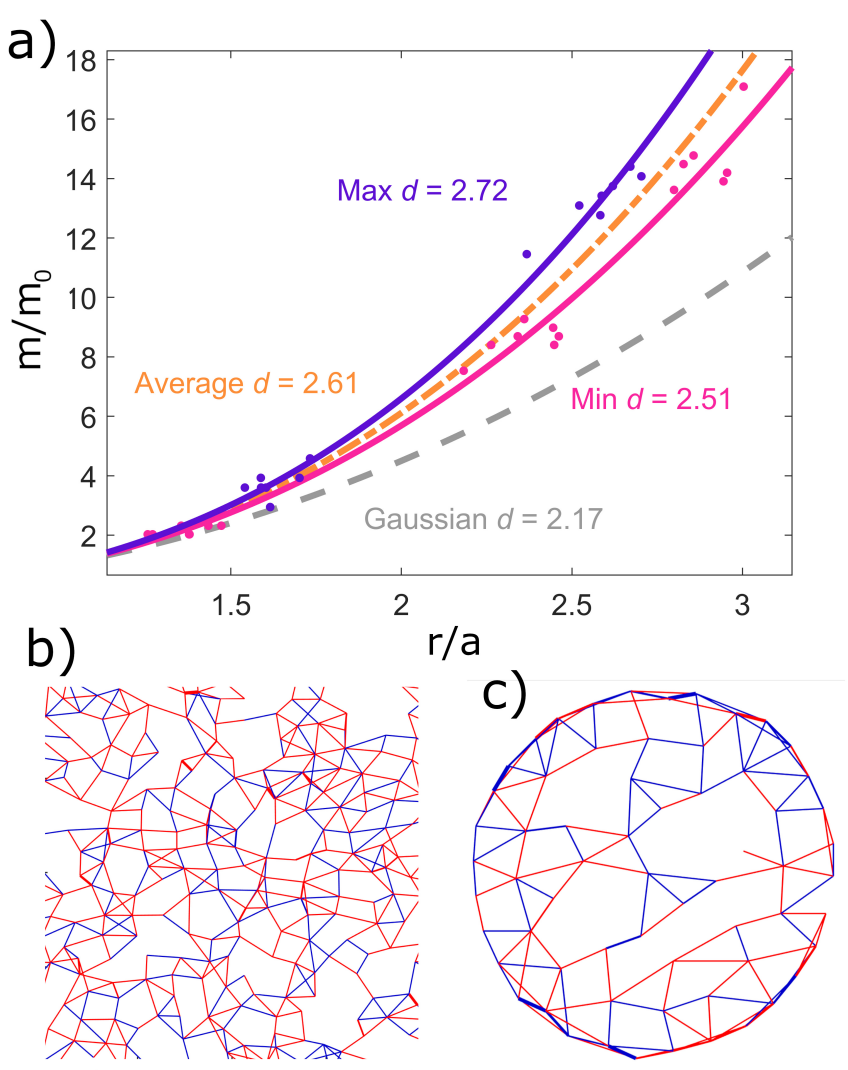

Fig. 4 Effective dimension of randomized magnetic systems. (a) $m(r)$ and its fits are plotted to demonstrate effective dimension $d$. To better compare between systems, each data point is divided by the $m_{0}$ determined by its fit. The purple and pink circles represent data from the Cayley trees with the largest $(d=2.72)$ and smallest $(d=2.51)$ effective dimensions, respectively, while the curves of corresponding color are their fits. The dashed curve represents the average effective dimension of all 21 investigated Cayley tree structures $(d=2.61)$. The dashed grey line corresponds to the effective dimension $(d=2.17)$ of a system with mere Gaussian disorder29. (b) and (c) Representative graphs of interactions for systems with Gaussian disorder and a typical Cayley tree, respectively.

proved in comparison to structures with Gaussian disorder 29 , as indicated by nearest-neighbor correlations that are closer to zero (see Fig. 33). As seen in Fig. 3b-c, this is manifested by a reduction of the ferromagnetic cluster size and regions of antiferromagnetic ordering. These results provide a first strong indication that such tree-like structures are better candidates in achieving artificial spin glasses. However, a finite non-zero spin glass transition temperature is still an obstacle that needs to be overcome 29131 . One strategy moving towards that goal relies on elevating the effective dimensions of such disordered structures, which is discussed in the following subsection.

\subsection{Effective dimension}

The dipolar Cayley trees discussed here, as any other artificial frustrated system, can be viewed as a network that consists of nodes (nanomagnets) and their connections (dipolar coupling between nanomagnets). While this network is embedded in two-dimensional space, inter-nanomagnet connections via dipo- 
lar coupling go beyond neighboring nanomagnets. As we will explain here, this can be used to elevate the effective dimension 30 . The notion of an effective network dimension may be understood in terms of how "scale" and "mass" relate to one another in different dimensions. One could consider a hypothetical shape existing in an arbitrary dimension, $d^{\prime}$. Its size may be changed by altering a length scale $r^{\prime}$ (the side length of a cube or a golden rectangle, for example). How does mass, or some other quantity present in the space, encompassed by this shape relate to this scale? If the density is constant and the dimension is an integer, the answer is simple. $m^{\prime}\left(r^{\prime}\right)=m_{0}^{\prime} r^{\prime d^{\prime}}$ where $m^{\prime}\left(r^{\prime}\right)$ is the mass, $m_{0}^{\prime}$ is the mass of the shape when $r^{\prime}=1, r^{\prime}$ is the scale of that shape, and $d^{\prime}$ is the dimension. Because $d^{\prime}$ is the exponent, we can tell that larger dimensions allow access to more "stuff" (mass, charge, magnetic moments etc.) with less scale. If the density is not constant or there are different ways of leaping from place to place, more or less stuff may be accessed than what is considered typical for dimension $d^{\prime}$. In these more complex systems, $d^{\prime}$ is replaced with $d$ and is allowed to vary as a real number, taking on the name "effective dimension"30. This definition allows the comparison of theoretical models on non-integer dimensions to real models with non-integer effective dimensions 30 . In spin glass models, the spin glass transition temperature depends on the effective dimension and for a critical dimension of $d_{c}=2.520$ the spin glass transition temperature has been predicted to be finite 39 . We will compare the effective dimension of our systems to this critical value to assess their viability as spin glasses.

To calculate the effective dimension of our experimental spin systems, a spin $i$ corresponding to a point $\mathbf{r}_{l=0}$ was chosen. $l$ labels the degree of separation from this spin. All spins connected to spin $i$, those with coupling strength $J_{i j}$ exceeding $20 \%$ the maximum strength, were accessed. The average distance between connected spins and the initial spin, $r_{l=1}$, was calculated. This can be considered the "scale" of the network's "shape." The total number of spins with this proximity, $m\left(r_{l=1}\right)$ (in analogy to the mass of a shape), were counted. This was repeated for further steps away from the center $(l=2$ through 5 , see the colored dots in Fig. 4 1 ). The process was reiterated for all spins $i$ less than $1.3 \mu \mathrm{m}$ from the center of the lattice to avoid edge effects. The resulting data were fit to $m(r)=m_{0} r^{d}$ via linear regression to determine $d$, the effective dimension (see curves of best fit and text in Fig. 4a). Notably, a true Cayley tree's density of nodes would exponentially grow from the origin, thus making the effective dimension infinite. In our case, the effective dimensions of the finite-sized Cayley trees exceed that of previously studied systems with Gaussian disorder $29, d=2.17$ (see Fig. 4 a) and the critical dimension $d_{c}=2.520$. They exhibit average effective dimension of 2.61 and a maximum $d=2.72$ (see Fig. 4 ). Fig. 4 p-c shows that the Cayley tree interaction structures expand this effective dimension with their tree-like arrangement.

Although the elevated spin glass transition temperature is not observable directly, we simulated the fabricated systems with the Monte Carlo method to probe their low-temperature behavior. A comparison of their field cooled and zero field cooled magnetic moments indicated that these systems could transition to a spin glass phase below $12.8 \mathrm{~K}$ (see Fig. S1). This is far below the freezing temperature of these nanomagnets but non-zero as anticipated. We conclude that a prospective extended structure built on an interaction concept similar to Fig. 45 could have the appropriate effective dimension and balance of competing interactions that would allow the realization of the first artificial Ising spin glass.

\section{Conclusions}

Despite the inherent difficulties in building tree-like interaction networks in two dimensions, our dipolar Cayley tree structures overcome previous limitations that hinereded the achievement of artificial spin glass systems 29 . However, not all randomly generated samples possess the same physics because of variations in the balancing of ferro- and antiferromagnetic interactions. The finite size of our samples produces fluctuations from average ordering (black triangles in Fig. 3a), allowing individual samples to exhibit more ideally balanced ordering $\left([C]_{N} \approx 0\right.$, see blue circles in Fig. 3a). This tunability is a substantial improvement over ordering in systems with Gaussian disorder 29 (grey squares in Fig 3a). Though less markedly variable, the effective dimension of the Cayley trees is also tunable, ranging from just below $d_{c}$ at $d=2.51$ all the way to $d=2.72$. Even though long range interactions break the perfect Cayley tree structure (see Fig. 4r), its dimension almost always remains high enough to allow a finite spin glass transition temperature. By contrast, the purely Gaussian-disordered system 29 did not alter its interactions into a network with high enough effective dimension (Fig. 4b), so that a finite-temperature spin glass phase could be accessed.

This work has taken two outstanding problems, unbalanced ordering and low effective dimension, and condensed them into one: making a larger Cayley tree system. This clear next step would allow for a raised spin glass transition temperature and novel, direct observation of spin glass dynamics via XMCD imaging. By simulating combinations of Cayley trees before they are fabricated, they may be vetted for balanced ordering and high effective dimension. Individual trees could then be combined as building blocks of a larger system, similar to how information is passed between logic gates in nanomagnetic computing 40. To further raise the transition temperature, interaction strength of the system could be augmented in comparison to the blocking temperature of the nanomagnets through stronger dipolar coupling. This future work would use model systems to bridge the gap between spin glass theory and experiment by exploring nonequilibrium thermodynamics of the spin glass phase.

\section{Conflicts of interest}

There are no conflicts to declare.

\section{Acknowledgements}

The authors would like to thank A. P. Young for fruitful discussions. This work was supported by the Swiss National Science Foundation (projects 174306 and 172774) and the Academy of Finland (project 316857). Part of the work was performed at the SIM beamline at the Swiss Light Source (SLS), Paul Scherrer Institute, 5232 Villigen, Switzerland. 


\section{Notes and references}

1 M. J. Harris, S. T. Bramwell, D. F. McMorrow, T. Zeiske and K. W. Godfrey, Physical Review. Letter 79, 2554 (1997).

2 M. Fu, T. Imai, T.-H. Han, and Y. S. Lee, Science 350, 655 (2015).

3 C. Castelnovo, R. Moessner, and S. L. Sondhi, Nature 451, 42 (2008).

4 A. Farhan, M. Saccone, C. F. Petersen, S. Dhuey, R. V. Chopdekar, Y.-L. Huang, N. Kent, Z. Chen, M. J. Alava, T. Lippert, A. Scholl, S. van Dijken, Science Advances 5, eaav6380 (2019).

5 K. Binder and A. P. Young, Review of Modern Physics 58, 801 (1986).

6 J. A. Mydosh, Reports on Progress in Physics 78, 052501 (2015).

7 N. Sourlas, Nature 339, 693 (1989).

8 S. Kirkpatrick, C. D. Gelatt Jr., M. P. Vecchi, Science 220, 671 (1983).

9 I. Recio, J. J.Torres, Neural Networks 84, 91 (2016).

10 A. G. Hudetz, C. J. Humphries, and J. R. Binder, Frontiers in systems neuroscience 8, 234 (2014).

11 A. Farhan, P. M. Derlet, A. Kleibert, A. Balan, R. V. Chopdekar, M. Wyss, L. Anghinolfi, F. Nolting, and L. J. Heyderman, Nature Physics 9, 375 (2013).

12 J. M. Porro, A. Bedoya-Pinto, A. Berger, and P. Vavassori, New Journal of Physics 15, 055012 (2013).

13 A. Farhan, P. M. Derlet, L. Anghinolfi, A. Kleibert, and L. J. Heyderman, Physical Review B 96, 064409 (2017).

14 I. Gilbert, G.-W. Chern, S. Zhang, L. O’Brien, B. Fore, C. Nisoli, and P. Schiffer, Nature Physics 10, 670 (2014).

15 Y. Perrin, B. Canals, N. Rougemaille, Nature 540, 410 (2016).

16 A. Farhan, A. Scholl, C. F. Petersen, L. Anghinolfi, C. Wuth, S. Dhuey, R. V. Chopdekar, P. Mellado, M. J. Alava, and Sebastiaan van Dijken, Nature Communications 7, 12635 (2016).

17 B. Canals, I.-A. Chioar, V.-D. Nguyen, M. Hehn, D. Lacour, F. Montaigne, A. Locatelli, T. Onur Menteş, B. Santos Burgos, and N. Rougemaille, Nature Communications 7, 11446 (2016).

18 J. Drisko, T. Marsh, and J. Cumings, Nature Communications 8, 14009 (2017).

19 A. Farhan, C. F. Petersen, S. Dhuey, L. Anghinolfi, Q.-H. Qin, M. Saccone, S. Velten, C. Wuth, S. Gliga, P. Mellado, M. J. Alava, A. Scholl, and S. van Dijken, Nature Communications 8, 995 (2017).

20 P. Andriushchenko, Journal of Magnetism and Magnetic Materials 476, 284 (2018).
21 M. Saccone, K. Hofhuis, Y.-L. Huang, S. Dhuey, Z. Chen, A. Scholl, R.V. Chopdekar, S. van Dijken, and A. Farhan, Physical Review Materials 3, 104402 (2019).

22 D. Shi, Z. Budrikis, A. Stein, S. A. Morley, P. D. Olmsted, G. Burnell, and C. H. Marrows, Nature Physics 14, 309 (2018).

23 V. Brajuskovic, A. Addi, C. Phatak, and A. K. Petford-Long, Physical Review B 98, 094424 (2018).

24 J. Sklenar, Y. Lao, A. Albrecht, J. D. Watts, C. Nisoli, G.-W. Chern, and P. Schiffer, Nature Physics 15, 191 (2019).

25 R. Macêdo, G. M. Macauley, F. S. Nascimento, and R. L. Stamps, Physical Review B 98, 014437 (2018).

26 D. Y. Lee and P. Tierno, Physical Review Materials 2, 112601(R) (2018).

27 R. A. Stancioli and L. A. S. Mól, Physical Review B 100, 024432 (2019).

28 C. F. Petersen, A. Farhan, S. Dhuey, Z. Chen, M. J. Alava, A. Scholl, and S. van Dijken, Applied Physics Letters 112, 092403 (2018).

29 M. Saccone, A. Scholl, S. Velten, S. Dhuey, K. Hofhuis, C. Wuth, Y.-L. Huang, Z. Chen, R. V. Chopdekar, and A. Farhan, Physical Review B 99, 224403 (2019).

30 L. Daqing, K. Kosmidis, A. Bunde, and S. Havlin, Nature Physics 7, 481 (2011).

31 A. K. Hartmann and A. P. Young, Physical Review B 64, 180404(R) (2001).

32 A. Farhan, A. Kleibert, P. M. Derlet, L. Anghinolfi, A. Balan, R. V. Chopdekar, M. Wyss, S. Gliga, F. Nolting, and L. J. Heyderman, Physical Review B 89, 214405 (2014).

33 M. Ostilli, Physical Review A 391, 3417 (2012).

34 D. J. Thouless, Physical Review Letters 56, 1082 (1986).

35 J. M. Carlson, J. T. Chayes, L. Chaves, J. P. Senthna, and D. J. Thouless, Europhysics Letters 5, 355 (1988).

36 M. Mézard and G. Parisi, The European Physical Journal B 20, 217 (2001).

37 L. Le Guyader, A. Kleibert, A. Fraile Rodríguez, S. El Moussaoui, A. Balan, M. Buzzi, J. Raabe, and F. Nolting, J. Elec. Spec. Rel. Phenom. 185, 371 (2012).

38 J. Stöhr Y. Wu, B. D. Hermsmeier, M. G. Samant, G. R. Harp, S. Koranda, D. Dunham, and B. P. Tonner, Science 259, 658 (1993).

39 M. Demirtaş, A. Tuncer, and A. N. Berker, Physical Review E 92, 022136 (2015).

40 H. Arava, N. Leo, D. Schildknecht, J. Cui, J. Vijayakumar, P. M. Derlet, A. Kleibert, and L. J. Heyderman, Physical Review Applied 11, 054086 (2019). 